\title{
Numerical modeling of ultrasonic particle manipulation for microfluidic applications
}

\author{
Süleyman Büyükkoçak • Mehmet Bülent Özer • \\ Barbaros Çetin
}

Received: 12 September 2013/Accepted: 7 April 2014/Published online: 22 April 2014

(C) Springer-Verlag Berlin Heidelberg 2014

\begin{abstract}
A numerical simulation methodology for ultrasonic particle/cell separation and cell washing processes is introduced and validated by comparing with the results from the literature. In this study, a finite element approach is used for modeling fluid flow in a microchannel and analytical relations are utilized for the calculation of the ultrasonic radiation forces. The solutions in acoustic and fluidic domains are coupled, and the particle separation under the influence of ultrasonic waves is numerically simulated. In order to simulate the cell washing process, diffusion and fluid dynamics solutions are coupled and solved. A Monte Carlo approach is chosen where statistical distributions are implemented in the simulations. Uniform distributions for the starting locations of particles/cells in the microchannel and normal distributions for the size of the particles are used in numerical simulations. In each case, 750 particles are used for the simulation, and the performance of separation process is evaluated by checking how many microparticles resulted in the targeted outlet channels. Channel geometries for the numerical simulations are adapted from the experimental studies in literature, and comparison between the reported experimental
\end{abstract}

S. Büyükkoçak · M. B. Özer

Department of Mechanical Engineering, TOBB University

of Economics and Technology, Ankara 06560, Turkey

e-mail: sbuyukkocak@etu.edu.tr

M. B. Özer

e-mail: bulent.ozer@gmail.com

B. Çetin $(\bowtie)$

Microfluidics and Lab-on-a-chip Research Group,

Mechanical Engineering Department, İhsan Doğramacı

Bilkent University, Ankara 06800, Turkey

e-mail: barbaros.cetin@bilkent.edu.tr results and the numerical estimations is performed. It has been observed that the numerical estimations and experimental results from the literature are in good agreement, and the proposed methodology may be implemented as a design tool for ultrasonic particle manipulation for microfluidic applications.

Keywords Microfluidics - Acoustophoresis · Particle separation - Acoustic radiation force . Acoustic standing wave

\section{Introduction}

During last decade, there has been an increased interest in the use of ultrasonic waves in microchannels to manipulate/separate particles or cells. The manipulation, separation and washing of particles and cells have several clinical and therapeutic applications in medicine and biology. Manipulation, separation and washing of cells are used for apheresis, stem and rare cell collections as well as buffer exchange applications for protection and storage of cells. Ultrasonic separation technology is one of the candidate technologies which may replace the use of centrifugation or membrane filtration technologies for the aforementioned clinical and therapeutic applications. The working principle relies on the generation of ultrasonic standing waves in a microchannel which results in an ultrasonic radiation force. Amplitude of the radiation force changes depending on the size of the particle, density of the particle and the speed of sound propagation within the particle. The difference in the amplitude of the radiation force results in particles of different properties (such as size or material) moving into different locations within a microchannel. Particles can be channeled out from these locations which 
lead to separation of particles from each other or the suspension medium.

It is possible to group the ultrasonic standing wave applications into two main groups: particle/cell position manipulation and particle/cell separation. The first group of applications aims to manipulate the cells or particles into certain locations along the microchannel (Neild et al. 2007; Townsend et al. 2004; Haake et al. 2005; Limaye and Coakley 1998; Hawkes et al. 1998; Dron et al. 2009; Nam et al. 2011). If the particle can be manipulated into certain locations inside the channel, this allows cell washing and cell concentration applications. Cell washing is exchanging the suspension fluid in which the cells are suspended, and cell concentration is increasing the number of cells per unit volume. Cell washing (Hawkes et al. 2004; Petersson et al. 2005b) and cell concentration (Evander et al. 2007; Bazou et al. 2012) have been shown to be successfully performed using ultrasonic standing waves in a microchannel. The second group of applications of ultrasonic standing waves is the particle (Adams and Soh 2010; Shi et al. 2009) and cell separation (Kumar et al. 2005; Petersson et al. 2005a, 2007). In these studies, microparticles with different sizes and cells of different types (such as different cell types in the blood) are separated from each other based on the differences in their size, density and/or acoustic properties.

The aforementioned studies mostly focused on the implementation of ultrasonic standing waves to certain applications through experiments. In the literature, the studies which show feasibility of this method through experiments are more common than the studies that focus on the numerical modeling of particle separation and manipulation. One reason for this is the difficulty of a complete numerical simulation of the problem due to multiple length scales associated with the computational domain and the different physical domains that should be coupled during the simulations. In a case where the ultrasonic waves are created using a piezoelectric material, a complete simulation of the separation problem requires modeling in electrical, mechanical, fluidic and acoustic domains along with a moving mesh due to the motion of the particles as well as simulations in time and frequency domains simultaneously. The limited number of studies in literature on the numerical simulation of this problem decreased the complexity of the problem with assumptions and simulated the ultrasonic particle/cell separation process under some assumptions. Glynne-Jones et al. (2013) used finite element method (FEM) to calculate the acoustic radiation force on an elastic sphere and elastic cylinder. In their study, acoustic radiation force is calculated using FEM, and it was found that the calculated results were in agreement with analytical solutions. Tripp et al. (2011) modeled the separation of lipid particles from erythrocytes through numerical modeling of fluidic domains (using finite volume technique) and analytical solution for the acoustic domain. They have designed an ultrasonic standing wave separator in the shape of a disk that enabled an axisymmetric model to be used for the analysis. This separator design was not tested experimentally, and the results were not compared with test results of this separator. Johnson and Feke (1995) designed a separator for microparticles. In their model, an analytical formulation for the ultrasonic radiation force was used. Fluid flow was modeled as a full-developed flow, and considering the straight geometry of the channel analytical solution for parabolic velocity profile was used in their model. The numerical model was also experimentally realized, and the results of the experimental measurements and the numerical models were found to be similar. Neild et al. (2006, 2007) considered an ultrasonic separator in which there is no fluid flow, but the particles align themselves on nodal pressure locations depending on the acoustic mode. FEM is implemented in their numerical model which modeled the piezoelectric material and the mechanical properties of the separator with static fluid inside the separator. Frequency domain analysis was performed to determine the number of nodal lines where the particles were aligned. These numerical predictions were validated with experimental results. A microparticle trap that operated at ultrasonic frequencies was modeled using FEM (Gralinski et al. 2012). The purpose of the study was to determine the size, number and the configuration of the piezoelectric material in order to optimize the performance of the ultrasonic particle-trapping device. In the study of Trujillo et al. (2013), the ramping of ultrasonic frequency was used to move the bands of the particles formed inside the device. A mass transport-based approach was used to model the particle movement. A one-dimensional FEM model was used which coupled the mass transport formulation with the acoustic radiation force.

In the present study, a numerical model of an ultrasonic standing wave-based separator and a cell washing microfluidic device is developed. This numerical model performs 3D fluid flow analysis using FEM, and the flow solution is coupled with the acoustic analysis as well as the diffusion analysis for the cell washing problem. In order for the numerical simulations to be realistic, a Monte Carlo type of approach is chosen which provides statistical distributions for the starting locations of particles as well as particle sizes for each microparticle size group. To the best of authors' knowledge, such statistics-based realistic simulation of the particle/cell washing and separation which couples the aforementioned domains does not exist in literature. All the numerical predictions are compared with the experimental results from the literature. For verification of the numerical results, the experimental setup which was introduced by Petersson et al. (2005b, 2007) is used. In the 
following section, the theory used in this study is provided. In Sect. 3, the approach implemented for the numerical modeling and the solution of the separation and washing processes is presented. In Sect. 4, simulation results and comparisons of the results with the experimental studies are given. In Sect. 5, the results are discussed, the verified model is used to understand the sensitivity of the separation performance to some of the input parameters and some recommendations for improving the performance are stated.

\section{Theory}

The calculation of the force generated on a particle due to an incoming acoustic pressure wave is called the acoustic radiation force, and a derivation of this force is given in the study of Yosioka and Kawasima (1955) and later by Gorkov (1962). The acoustic radiation force derivation starts from Navier-Stokes equations for an inviscid compressible fluid. For the solution of the Navier-Stokes equation, it is a common approach to assume that the acoustic input to the medium creates first-order harmonic perturbation on the medium density and pressure. However, this approach is not useful in calculation of the acoustic radiation force since the first-order perturbations average out to zero over one cycle. If the acoustic radiation force on the particle averages out to zero, the particle cannot have a net movement under the acoustic field. Considering the second-order perturbations, the acoustic radiation force acting on a micron-sized particle in an ultrasound field can be written as Settnes and Bruus (2012):

$\mathbf{F}_{\mathrm{rad}}=-\nabla U_{\mathrm{rad}}$,

where $U_{\text {rad }}$ is the radiation potential,

$$
\begin{aligned}
& U_{\mathrm{rad}}=\frac{4 \pi}{3} a^{3}\left[f_{1} \frac{1}{2 \rho_{f} c_{f}^{2}}\left\langle p_{\mathrm{in}}^{2}\right\rangle-f_{2} \frac{3}{4} \rho_{f}\left\langle\mathbf{v}_{\mathrm{in}}^{2}\right\rangle\right], \\
& f_{1}=1-\frac{\rho_{f} c_{f}^{2}}{\rho_{p} c_{p}^{2}}, f_{2}=\frac{2\left(\rho_{p}-\rho_{f}\right)}{2 \rho_{p}+\rho_{f}} .
\end{aligned}
$$

In the above formulation, $a$ is the radius of the spherical particle, $\rho_{p}$ is the density of the particle, $\rho_{f}$ is the density of the suspension fluid, $c_{p}$ is the speed of sound inside the particle, and $c_{f}$ is the speed of sound inside the suspension fluid. The Eqs. (1)-(3) show that the acoustic force applied on the particle is a function of particle properties. Size seems to be the property that affects the acoustic radiation force the most since the acoustic radiation force is proportional with $a^{3}$. The force and its direction also depend on the particles' density and the speed of sound inside the particle. The $p_{\text {in }}$ and $\mathbf{v}_{\text {in }}$ terms in Eq. (2) represent the incident acoustic pressure and the acoustic particle velocity without considering any scatter (Settnes and Bruus 2012). In practice, in order to maximize the incident pressure in the medium, resonance condition in a long narrow channel needs to be created. Generally, the frequency of the oscillation of the channel wall needs to be selected such that half the wavelength of the acoustic wave at that frequency equals to the channel width. This condition creates a standing wave along the $y$-direction (channel width). The velocity potential for the acoustic wave along the $y$-direction can be defined as follows:

$$
\phi(y, t)=\frac{1}{k} u_{w} \cos (k y) \cos (\omega t) .
$$

In Eq. $4, u_{w}$ denotes the wall velocity, $k$ denotes the wave number of the acoustic wave, and $\omega$ is the frequency of the oscillations ( $\mathrm{rad} / \mathrm{s})$. Using the incident velocity potential, one can calculate the incident acoustic pressure through time derivative and calculate acoustic particle velocity through spatial derivative of the velocity potential along $y$ direction. If the incident acoustic pressure and acoustic particle velocity are substituted in Eqs. (1) and (2), the following relationship for the acoustic radiation force along the width of a channel can be obtained as Gorkov (1962):

$F_{\mathrm{rad}}^{y}=4 \pi a^{2}(k a) E_{\mathrm{ac}} \Phi \sin (2 k y)$,

where

$\Phi=\frac{\rho_{p}+\frac{2}{3}\left(\rho_{p}-\rho_{f}\right)}{2 \rho_{p}-\rho_{f}}-\frac{\rho_{f} c_{f}^{2}}{3 \rho_{p} c_{p}^{2}}, E_{\mathrm{ac}}=\frac{1}{4} \rho_{f} u_{w}^{2}$.

In the simulations performed in this study, the acoustic radiation force is calculated using the Eqs. (4) and (5).

The forces that affect the particle movement are not only acoustic radiation forces, but also the drag forces on the particles due to the fluid flow. This study considers the separation of different size/density microparticles in a microfluidic network. Therefore, in order to analyze the particle trajectories, the flow field within the microfluidic network needs to be determined. The flow field is governed by the incompressible Navier-Stokes equation:

$\rho \mathbf{u} \cdot \nabla \mathbf{u}=-\nabla p+\mu \nabla^{2} \mathbf{u}$

where $\mathbf{u}$ is the fluid velocity, $p$ is hydrodynamic pressure, and $\mu$ is the dynamic viscosity of the fluid. Unlike the calculation of the acoustic radiation force, an analytic solution approach is not possible; therefore, a numerical approach is used and $3 \mathrm{D}$ fluid flow is simulated using COMSOL Multiphysics (COMSOL Inc., USA). COMSOL Multiphysics software is a FEM-based numerical simulation tool. Its ability to integrate with MATLAB software makes this software really suitable for this study. Once the velocity field is determined, the drag force experienced by 
the particle within the microchannel can be obtained using Stokes law due to low-Reynolds number nature of the fluid flow within the microchannel as:

$\mathbf{F}_{\text {drag }}=6 \pi \mu a\left(\mathbf{u}-\mathbf{u}_{p}\right)$,

where $\mathbf{u}_{p}$ is the particle velocity.

In the modeling of the particle flow, it is assumed that the inertial forces are negligible. This is an assumption that is justifiable for the particle size ranges (3-10 $\mu \mathrm{m}$ diameter) investigated in this study. It is also assumed that the forces due to Brownian motion are neglected in this study. This assumption is generally justified in literature for particles above one micron. Another assumption of this study is the neglection of the gravity-induced sedimentation of the particles. This is justified due to short residence times of the particles inside the channel ( $\sim 1-2 \mathrm{~s}$; Martinez-Duarte et al. 2010). As a result, only two forces are assumed to act on a sphere as illustrated in Fig. 1. Equating the drag force and the acoustic radiation force, particle position within the microchannel can be obtained as:

$y(t)=\frac{1}{k} \arctan \left[\tan [k y(0)] \exp \left(\frac{4 \Phi}{9}(k a)^{2} \frac{E_{\mathrm{ac}}}{\mu} t\right)\right]$

Equation (9) shows the trajectory of the particle, and $y(0)$ is the initial sphere starting position and $t$ is the exposure time to the acoustic field. The forces on particles and the trajectory of the particles in the separator geometry are shown in Fig. 1.

For cell washing purposes, it is critical to estimate the diffusion between the fluid in the side inlet channels (contaminated) and the fluid in the center inlet channel (clean). In order to define the diffusion process, the convective-diffusion equation is used:

$\mathbf{u} \cdot \nabla c=D \nabla^{2} c$,

where $c$ represents mole fraction of the buffer solution flowing from the central entry of channel, $\mathbf{u}$ is the fluid velocity obtained from the solution of Navier-Stokes equations, and $D$ is the diffusion coefficient for the two types of fluids used inside the microchannel.

\section{Methods for numerical solution}

The main steps of numerical solution procedure are given in the flowchart as shown in Fig. 2. In order for an acoustic field to be induced inside a microchannel, the channel walls need to be excited at an ultrasonic frequency. Commonly a piezoelectric material that is in contact with the outer walls of the channel is used for the excitation. This excitation also vibrates the inner part of the microchannel walls. In order to simulate this part of the problem, an electromechanical model of the piezoelectric material and the structural model of the channel are needed. There are several FEM-based softwares that can be used to simulate this part of the problem. However, the electro-mechanical part of the problem is not modeled in the current study. The scope of this study does not include how the walls are moved but rather is limited to modeling the physics as a result of this channel wall excitation. The omission of modeling of the piezoelectric actuator may affect the separation estimations. Mode shapes of the piezoelectric material affect the velocity and pressure distributions inside the channel (especially along the length direction). It is expected that the estimations may suffer from the absence of the modeling of the dynamics of the piezoelectric behavior (which affects the mode shapes). In Fig. 2, the simulation steps that are not considered in this study are drawn with dash lines.

The vibration displacement amplitude of the walls and the frequency at which they are excited enable one to determine the channel wall velocity. The wall vibration frequency is selected so that half the wavelength of the acoustic wave equals to the channel width. Using Eq. (6),

Fig. 1 Drawing of an ultrasonic particle separator

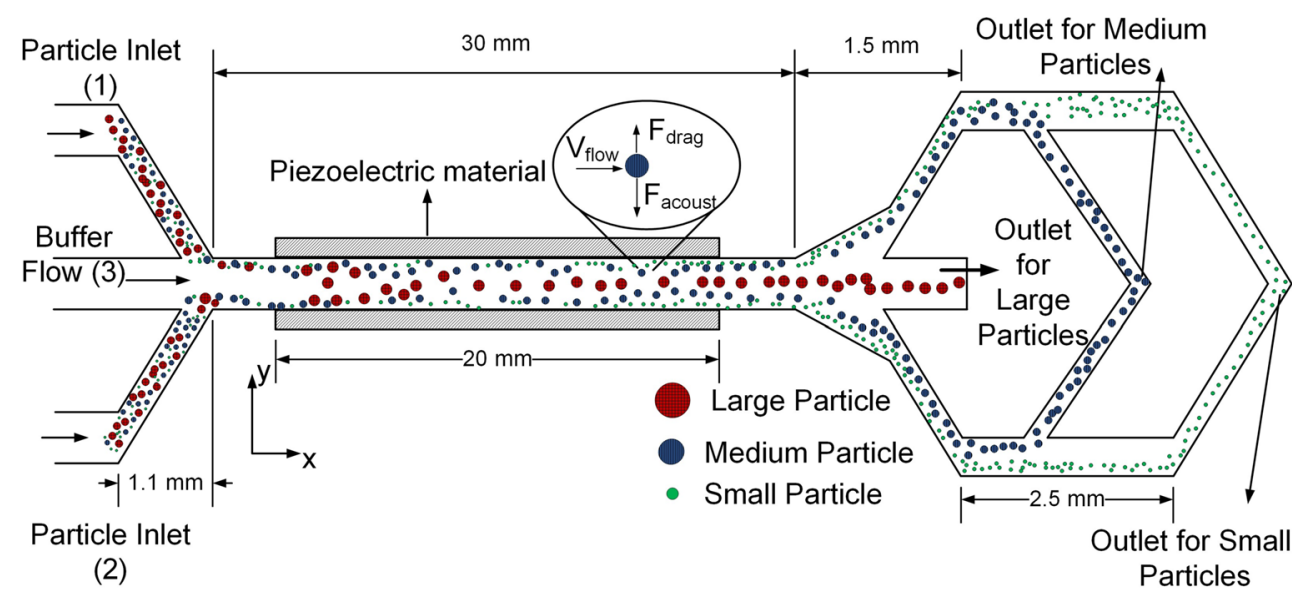


Fig. 2 Numerical simulation steps for particle/cell separation using acoustophoresis

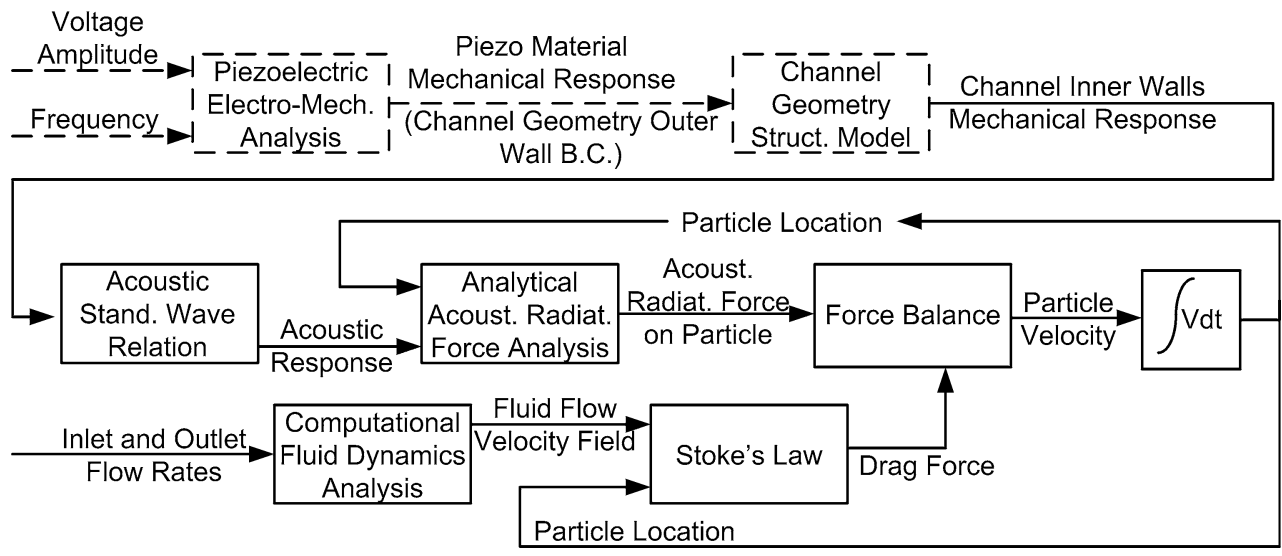

Table 1 Input parameters for the simulations

\begin{tabular}{ll}
\hline Density & $\rho=1,000 \mathrm{~kg} / \mathrm{m}^{3}$ \\
Viscosity & $\mu=0.9 \times 10^{-3} \mathrm{~kg} / \mathrm{ms}$ \\
Particle diameter & $d=3,7,10 \mu \mathrm{m}$ \\
Diffusion coefficient & $D=10^{-9} \mathrm{~m}^{2} / \mathrm{s}$ \\
Speed of sound in the fluid & $c=1,500 \mathrm{~m} / \mathrm{s}$ \\
Speed of sound in polystyrene & $c_{p} \approx 2,350 \mathrm{~m} / \mathrm{s}$ \\
& $($ Smith and Wiggins 1972) \\
Wave number & $k=84911 / \mathrm{m}$ \\
\hline
\end{tabular}

the $E_{\text {ac }}$ value can be calculated. Using Eqs. (5) and (6), the force due to the ultrasonic wave field can be predicted. It should be noted that the magnitude of the acoustic radiation force changes depending on the $y$-location of the particle along the channel width. During the simulation, 750 particles are used (equal numbers for each particle size group), and a normal distribution with a mean and a standard is assigned for the diameter of the each group. The acoustic force calculations of these 750 particles are performed in MATLAB software. One of the main assumptions in this study is the low concentration of particles. The formulation used to calculate the acoustic radiation force on each particle assumes that multiple reflections (reflections from other particles) do not occur. Once the multiple reflections are assumed to occur (due to high concentration of particles), one needs to model not only the fluid domain, but also the acoustic domain using FEM. This would increase the computational burden significantly, and the problem would be beyond computational processing power of the system used in this study. In the experimental study, a concentration of 50,000 particles/ $\mu \mathrm{l}$ was used by Petersson et al. (2007). The input parameters used in the numerical analysis are tabulated in Table 1 . The diffusion coefficient is taken as $1 \times 10^{-9} \mathrm{~m}^{2} / \mathrm{s}$ which is a typical value for aqueous solutions (Bhagat and Papautsky 2008).

As shown in Fig. 2, using the flow rates at the inlets and the outlets of the separator as the inputs to the computational fluid dynamics (CFD) model (performed in COMSOL), the steady flow field in the separator is calculated. The flow velocity response at three principle directions is exported to MATLAB, and at each particle location, velocity at a point is interpolated from this velocity field lookup table during the coupled simulations. Solving the force balance equation, the velocity of the particles due to the acoustic waves and flow conditions is determined in all three principle directions. Following the calculation of the velocity of the particles, a time step is taken (it is assumed that the velocity is constant during that time step), and the new positions of the particles are evaluated. Time steps can be adjusted to be constant or adaptive such that particles move equal distances during each time step. After the new position of the particles is computed, analysis steps are repeated in MATLAB software until the particle exits the separator from one of the three outlets of the separator. This is performed for 750 particles for each run. During the analysis, it is assumed that the particles do not disturb the flow field. The interactions between the particles such as collisions are also ignored. Both of these assumptions are valid if the particle concentration in the channel is low. There is also a check in the MATLAB code to ensure that the distance between the center of the particles and channel walls to be less than the radius of the particle.

\section{Results}

In the coming three sub-sections, the results of the numerical models which are used to simulate three different experimental studies from the literature are presented. The results of the actual experiments obtained from the literature are used to verify the numerical results. In Sect. 4.1 , the numerical modeling of an experimental study where the particles of different size groups were separated using ultrasonic standing waves is given. In Sect. 4.2, a 
numerical study which performs the simulation of an experimental setup in which microparticles are separated based on their densities is presented. In Sect. 4.3, the results for the numerical simulations of a particle washing experimental setup are presented. As aforementioned, a statistical approach is implemented in the simulations. Both size and starting location distributions are assigned for the particles. Statistical approach is critical to represent the real-life situation and obtain meaningful simulation results in terms of distributions (i.e., not a single value) which are comparable with the experimental results.

\subsection{Separation of particles with different sizes}

To illustrate the evaluation of performance of the acoustophoresis process with the proposed computational model, the performance of the device proposed by Petersson et al. (2007) is assessed. Figure 1 shows the channel geometry used for the numerical analysis which is the nonscaled 2D section of the actual 3D model. The width of the channel is $370 \mu \mathrm{m}$, and the height of the channel is $125 \mu \mathrm{m}$; hence, it is assumed that the acoustic field is uniform along the height of the channel. Microscale particles have mean diameters of 3,7 and $10 \mu \mathrm{m}$ and from the manufacturers' data sheet (http://www.polysciences.com/ Catalog/Department/81/categoryid-373/) of polystyrene particles with standard deviations of 5,10 and $15 \%$ of the mean diameters are used in the normal distribution, respectively (normal size distributions of the particle groups are illustrated in Fig. 10a with histogram plots and corresponding curve fits). Particles are released from Inlet 1 and Inlet 2 with uniform distribution along the cross sections of the side inlets (see Fig. 1). It can be seen from Fig. 3a that since the inlet flow rate at the side channel is significantly lower than the flow rate of the center channel, particles coming from the side channels are pushed toward the upper channel wall which causes a bubble shape appearance at the inlet junction, and at this point, particles are in mixed state. They enter the straight portion of the microchannel which is $3 \mathrm{~cm}$ long, but only $2-\mathrm{cm}$ portion is excited by the piezoelectric material generating acoustic waves at $2 \mathrm{MHz}$. As the particles move along the straight portion of the channel, they start to experience the acoustic radiation force which pushes them to the center of the channel. Acoustic radiation force is the largest on the particles with the largest diameter $(10-\mu \mathrm{m}$ particles). Therefore, these particles (shown in red in Fig. 3) are closest to the center of the channel and are separated out from Outlet 1 as shown Fig. 3b. A total of $3 \mu \mathrm{m}$ (yellow lines in Fig. 3) and $7 \mu \mathrm{m}$ (black lines in Fig. 3) particles are closer to the channel wall and do not exit from Outlet 1 but continue toward the side channel which leads to Outlet 2 and Outlet 3 as shown in Fig. 3b, c. Since $3-\mu \mathrm{m}$ particles
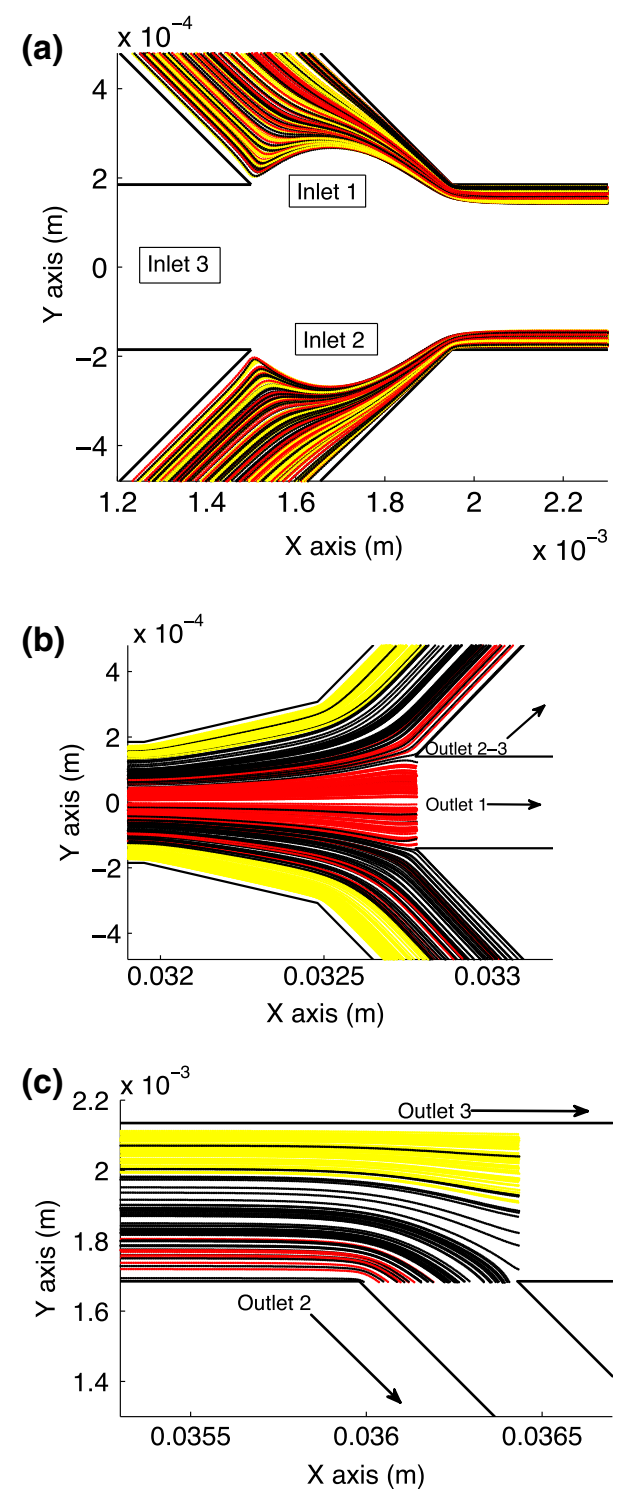

Fig. 3 Trajectories of particles (red $10-\mu \mathrm{m}$ particles, black $7-\mu \mathrm{m}$ particles, yellow $3-\mu \mathrm{m}$ particles): $\mathbf{a}$ at the inlet junction, $\mathbf{b}$ at the exit junction of main channel, $\mathbf{c}$ at the exit junction of the second and the third outlet (color figure online)

(yellow lines) are the smallest particles on which the acoustic radiation force is the weakest, they travel closest to the channel walls. At the junction of Outlet 2, negative pressure created by the pump is not large enough to attract them into Outlet 2. Hence, 3- $\mu$ m particles lead to Outlet 3, whereas the $7-\mu \mathrm{m}$ particles which are closer to the Outlet 2 junction are separated out (Fig. 3c). This separation process is shown schematically in Fig. 1, and the numerical simulation results for the separation process are shown in Fig. 3. The experimental flow rates reported in Petersson et al. (2007) which are $0.13 \mathrm{ml} / \mathrm{min}$ for each outlet and $0.04 \mathrm{ml} / \mathrm{min}$ for the side channels (i.e., sum of Inlet 1 and Inlet 2) are used for the fluid flow analysis. 


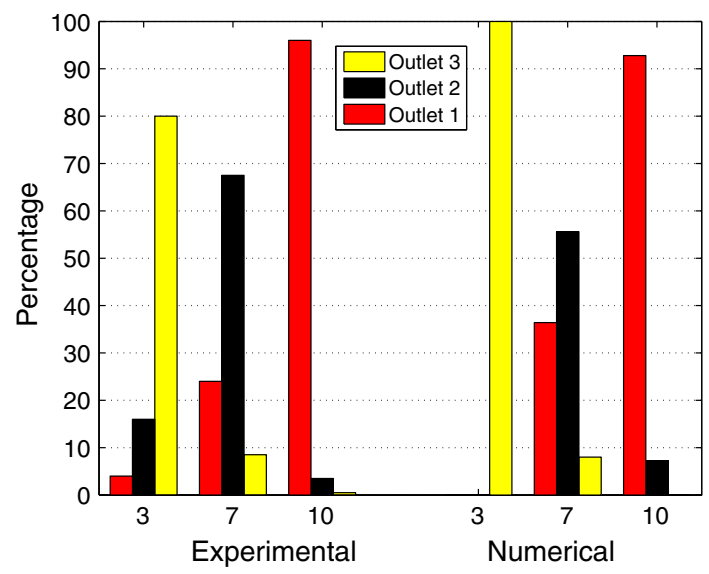

Fig. 4 Bar plot of experimental (adapted from Petersson et al. 2007) and numerical results

The experimental results reported in Petersson et al. (2007) are used for verification of the numerical results. The results of the separation experiments for the three sizes of polystyrene microparticles are regenerated in Fig. 4. It can be observed from the experimental results that similar to numerical predictions, the most efficiently separated particles are 3- and $10-\mu \mathrm{m}$-diameter particles. The numerical simulation predicts that only $55 \%$ of the $7-\mu \mathrm{m}$ particles exited from the targeted Outlet 2 , the experiment shows that $65 \%$ of $7-\mu \mathrm{m}$ particles were successfully separated. The numerical and experimental results for the separation efficiency of $10-\mu \mathrm{m}$ particles have similar results. The most significant deviation from experimental results was at the $3 \mu \mathrm{m}$ particle size. The numerical simulations predict perfect separation of these particles; however, the experiments show that the actual separation efficiency is $80 \%$. It is not believed that this is due to the calculation of acoustic radiation force lower than it actually is. If this was the case, the numerical predictions should have larger errors on the 7- and $10-\mu \mathrm{m}$ particles which were not the case. In the study of Petersson et al. (2005a), it was mentioned that as the velocity of the particles increase, the efficiency of the cell washing seemed to become lower. It was mentioned in their study that one possible reason for this might be the transport of contaminated fluid by the particles as they move rapidly toward the center of the channel. Similarly, it may be possible that the smaller particles are carried away by larger particles (volume ratio between 10 - and $3-\mu \mathrm{m}$ particles is roughly 30 times) toward the center of the channel. Since it is assumed that there are no collisions between the particles such an event cannot be captured by the proposed numerical model; hence, the hypothesis about smaller particles being carried away could not be verified. Even with the difference of $20 \%$ in $3-\mu \mathrm{m}$ particle separation estimate, generally the results are in good agreement with the experiments and the

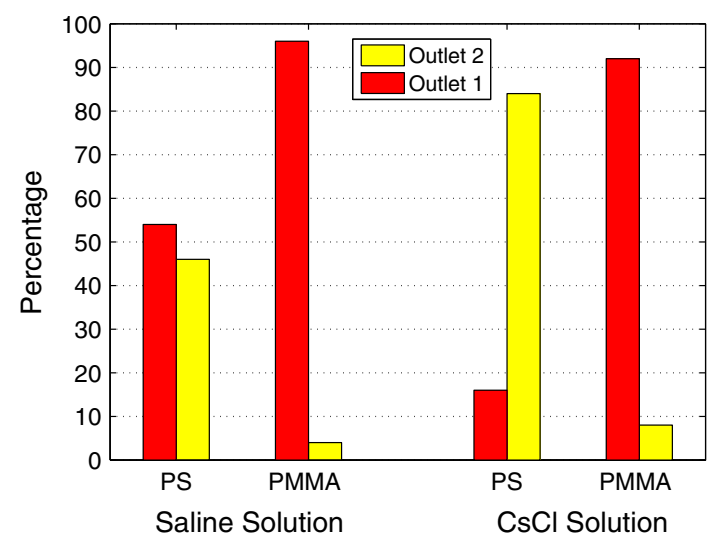

Fig. 5 Numerical results for the separation in different solutions

general trends for the separation of each particle are successfully captured. It is also possible the discrepancy between the numerical and experimental results may be due to the uncertainties and difficulties in the execution and measurements during the experimental study, as well as uncertainties associated with the fabrication of the fluidic chip.

\subsection{Separation of particles with different densities}

Separation of particles with the same size but different densities is also simulated. In the previous study by Petersson et al. (2007), the separation of $3-\mu \mathrm{m}$ polystyrene particles (density $1.05 \mathrm{~g} / \mathrm{cm}^{3}$ ) and 3- $\mu$ m PMMA particles (density $1.22 \mathrm{~g} / \mathrm{cm}^{3}$ ) on the aforementioned ultrasonic separator test setup was performed. In this experiment, only two outlets of the separator shown in Fig. 1 were used. A numerical simulation study is performed based on their test setup. The results of separation simulations in saline solution $\left(0.9 \% \mathrm{NaCl}, 1004.6 \mathrm{~g} / \mathrm{cm}^{3}\right)$ for the polystyrene and PMMA particles are shown in Fig. 5. It can be observed that the particles cannot be separated in saline solution. An optimal value for the $E_{\text {ac }}$ value was searched for the separation of polystyrene and PMMA, even with the best $E_{\text {ac }}$ value, almost all of the PMMA and half of the polystyrene particles exit through the same channel (Outlet 1). This is due to the closeness of the contrast factors [shown in Eq. (6)] of the two particle types in a saline solution. The acoustic force is determined by the contrast factor which needs to be significantly different for the two different types of particles for a successful separation.

The next step was to change the medium density such that a significant difference can be achieved for the two contrast factors. In the study of Petersson et al. (2007), cesium chloride $(\mathrm{CsCl})$ was added to the saline solution until the density of the buffer medium became $1.16 \mathrm{~g} / \mathrm{cm}^{3}$. After increasing the density of the buffer medium, the 
numerical results for separation are shown in Fig. 5 (right side). It can be observed from the figure that $\sim 93 \%$ of PMMA particles exit through Outlet 1 and $\sim 85 \%$ of polystyrene particles exit through Outlet 2 which shows significant improvement compared to the results of the saline solution.

In the experimental study of Petersson et al. (2007), similar to results shown in Fig. 5, it was reported that the separation was not successful when the particles were suspended in saline solution. After $\mathrm{CsCl}$ was added to the buffer medium which increased the density of the suspension fluid, it was reported that $96 \%$ of the PMMA particles exited from Outlet 1 and $88 \%$ of the polystyrene particles exited from Outlet 2. The numerical solution, as shown in Fig. 5, estimates that $\sim 85 \%$ of the polystyrene and $\sim 93 \%$ of the PMMA particle leave the separator from the intended channels. Therefore, for particle washing simulations, the numerical simulations predict the experimental results quite accurately.

\subsection{Particle washing}

This part of the study focuses on the numerical modeling of fluid exchange in the separator. In the process of particle washing, particles are suspended in the contaminated fluid at the inlet location, and they enter the separator from the inlet side channels (particle inlets of Fig. 1). The clean buffer is fed into the separator from the center inlet channel (buffer inlet of Fig. 1). Since the flow is a low-Reynolds number flow, the mixing between the fluids is mainly due to the diffusion process. The particles along the length of the channel are subjected to acoustic radiation force and move to the center of the channel where the clean buffer flows. This process results in switching of the fluid medium for particles. The feasibility of this concept was shown experimentally in Petersson et al. (2005b). Numerical modeling of the particle washing is performed by coupling the fluidic, diffusion and acoustics domains in COMSOL Multiphysics and MATLAB environment. In the experimental model, a geometry similar to the separator geometry shown in Fig. 1 is used. The main difference between the geometry used for the washing and separation is the different number of outlet channels. In the particle washing case, the geometry used has only three branches at the outlet. No further branching occurs after the initial branching at the Outlet 1 location. In FEM model, only the quarter of the channel network is modeled due to symmetry.

In Figs. 6 and 7, the results of coupled fluid flow and diffusion analysis are shown. The flow rates used in the analysis are indicated on the graphs which are the reported flow rates in Petersson et al. (2005b). The values of the relative concentrations correspond to colors in the graphs.
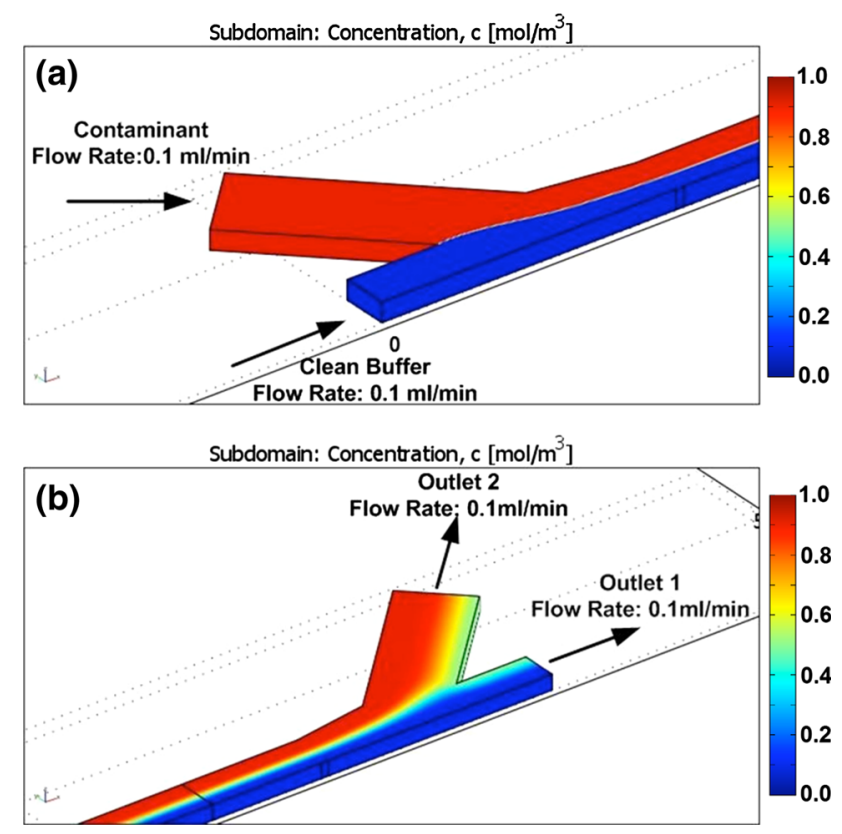

Fig. 6 Concentration field within the particle wash channel: $\mathbf{a}$ at the inlet, $\mathbf{b}$ at the outlet
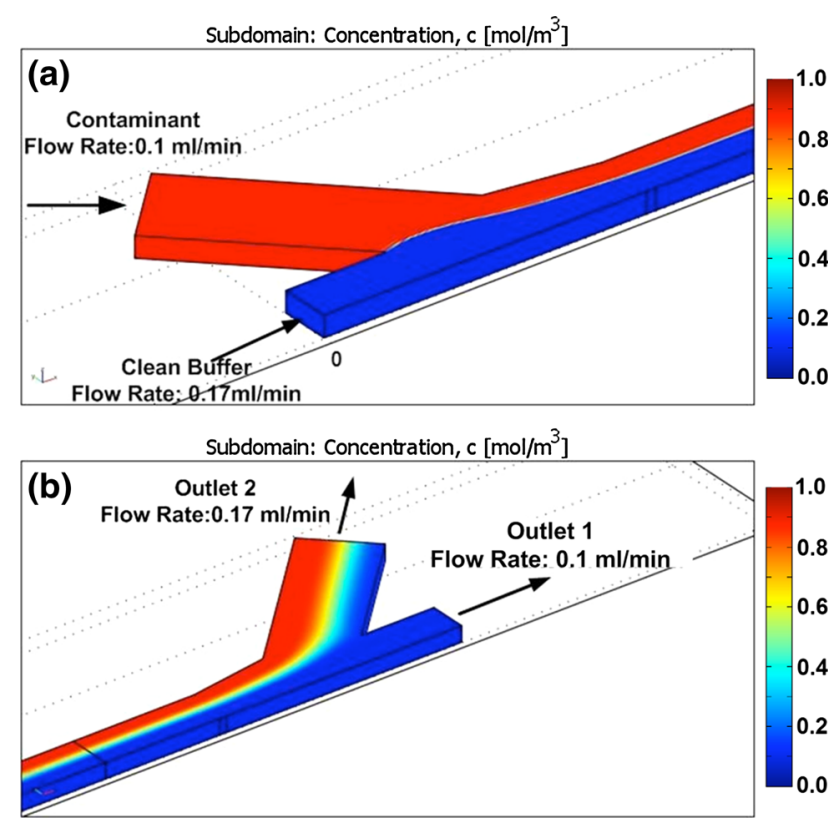

Fig. 7 Concentration field within the particle wash channel (Case 2): $\mathbf{a}$ at the inlet, $\mathbf{b}$ at the outlet

The contaminant concentration is taken as concentration value of unity, and the concentration of the clean buffer is taken as zero. Figure $6 a$ shows the starting concentration at the inlet, and how the two fluids of different concentration merge at the inlet of the washing channel. Figure $6 \mathrm{~b}$ shows the fluid concentration at the outlet region. It can be seen from the figure that some portion of the contaminant is flowing at the center outlet where the particles are located 
which is not a desired situation. Therefore, in order to improve the washing performance in Petersson et al. (2005b), the initial flow rates at the center inlet and side outlet are increased. The flow rates at the remaining two branching locations are left to be the same (Fig. 6). The results after the change in flow rates are shown in the plots of Fig. 7b. It can be observed that the center outlet channel (where the particles exit from) has the clean buffer dominantly.

Another important criterion is not to lose significant amount of particles from the side outlet channels. This becomes even more important for Case 2 since the flow rate of Outlet 2 was increased to minimize the exit of the contaminated fluid from the center outlet. In order to investigate the trajectories of the particles, the acoustic analysis is also added onto the fluid dynamics-diffusion coupled simulation of the previous case study. Here, only one type of microparticles which has a nominal diameter of $5 \mu \mathrm{m}$ with a standard deviation of $10 \%$ of the nominal diameter is used. In order to keep the visual appearance clear, 67 microparticle trajectories are drawn. As shown in Fig. 8a, the particle trajectories (black lines) start in the red (contaminated) fluid. Due to acoustic radiation, force particles move toward the center and they exit the wash channel from the center outlet in the blue (clean) fluid as

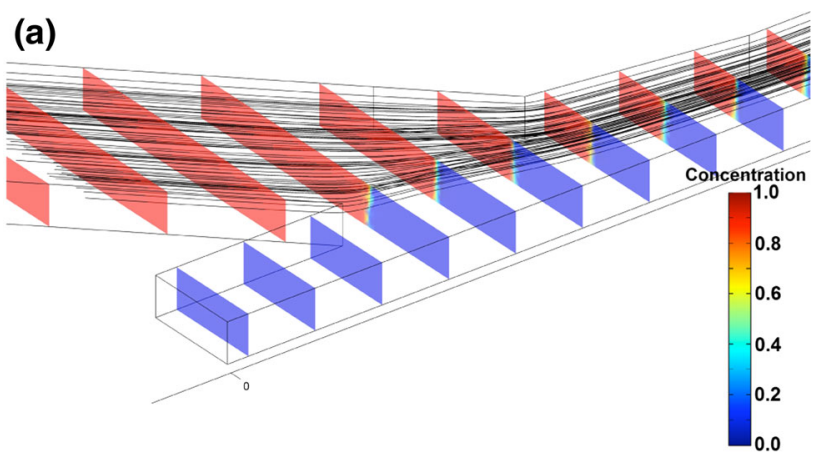

(b)

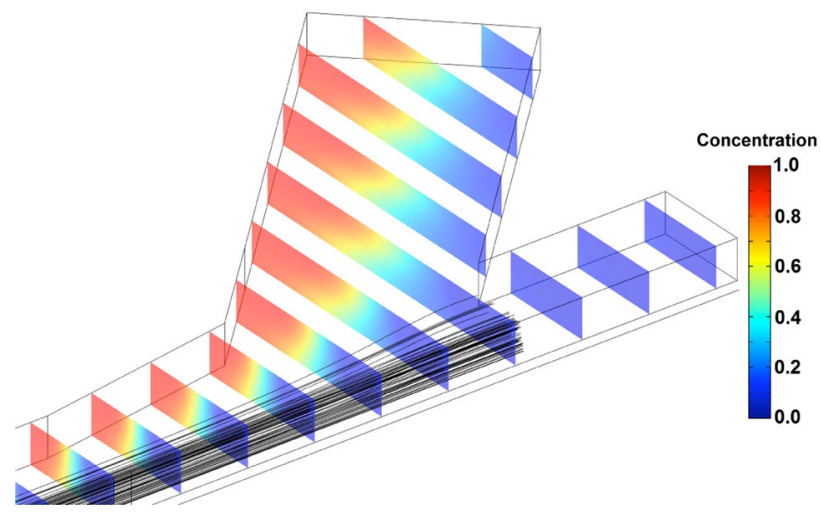

Fig. 8 Particle trajectories (Case 2): a at the inlet of the particle wash channel, $\mathbf{b}$ at the outlet of the particle wash channel shown in Fig. 8b. Loss of microparticles to the side channel during washing is not observed.

In order to verify the numerical results for particle washing, results of the experimental study by Petersson et al. (2005b) are used. The geometry and the flow rates of the numerical study were chosen to match the separator and the flow rates in the experimental study. The first numerical case study in this section has equal flow rate of $0.10 \mathrm{ml} /$ min at each inlet and outlet. The mixing of the contaminated fluid into the clean buffer outlet (as shown in Fig. 6b) was also observed in Petersson et al. (2005b). After the modification of flow rates (as shown in Fig. 7), it is mentioned in Petersson et al. (2005b) that a clean medium buffer zone in the side outlet channel was observed which is clearly visible in the numerical results shown in Fig. 7b. So, it can be seen that, descriptively, the numerical results and the experimental observations match. However, a numerical comparison enables to draw a more quantified comparison between the numerical and experimental results. In order to quantify the effectiveness of the cell washing process in Petersson et al. (2005b), a parameter called medium exchange efficiency was introduced. It shows the fraction of the contaminant that leaves from the side outlets. In order to calculate this parameter in the numerical simulations, the concentration of the contaminant that enters the washing channel in unit time is calculated. Then, the concentration of contaminant that leaves from the side outlet in unit time is calculated, and these two numbers are divided. If the ratio is unity, it means that the washing system removes all of the contaminant fluid from the side outlet. The average concentrations are calculated by taking the volume integral of concentration shown in Figs. 6 and 7. The calculated and experimental medium exchange efficiency values are given in Table 2.

The medium exchange efficiency results reported in Petersson et al. (2005b) are given at different voltage amplitudes applied on the piezoelectric material. As the voltage amplitude increases, the particles move more rapidly toward the center of the channel where the particles may carry some amount of the contaminated fluid during their movement. The transfer of the fluid due to movement of the particles is not modeled in the FEM; therefore, the experimental results with the lowest reported excitation voltage values are chosen for the comparison. Table 2 shows that the computational estimates of efficiencies seem slightly higher than the experimental ones. One possible reason for this may be due to not accounting the drag of the small amount of contaminant fluid surrounding the particle in the numerical model. However, there is still good agreement with the numerical and experimental medium exchange efficiencies. It is shown that the numerical simulation tool can be used to estimate the experimental washing performance for the reported flow rates. 
Table 2 Comparison of experimental and numerically calculated medium exchange efficiency values

\begin{tabular}{llc}
\hline & $\begin{array}{l}\text { Exp.* } \\
(\%)\end{array}$ & $\begin{array}{l}\text { Num. } \\
(\%)\end{array}$ \\
\hline $\begin{array}{l}\text { Medium exchange efficiency (Case } 1 \\
\text { with } 0.10 \mathrm{ml} / \text { min flow rate at the side outlet) }\end{array}$ & $81^{\dagger}$ & 83 \\
$\begin{array}{l}\text { Medium exchange efficiency (Case } 1 \\
\text { with } 0.17 \mathrm{ml} / \text { min flow rate at the side outlet) }\end{array}$ & $89^{\dagger}$ & 93 \\
$*$ From Petersson et al. (2005b) with $1.5 \%$ particle concentration \\
$+6 \mathrm{~V}_{\mathrm{pp}}$ actuation voltage \\
$* 10 \mathrm{~V}_{\mathrm{pp}}$ actuation voltage
\end{tabular}

Therefore, it may be used as a design tool to optimize the performance of washing procedure around the flow rates reported in Table 2.

\section{Discussion}

It has been shown that the proposed numerical methodology can predict the fluid and particle flow within a microfluidic device, so far. In this section, some discussions about the effects of flow rate, starting locations of the particles and the particle size distribution on the separation performance are provided and some suggestions for performance improvements are proposed.

\subsection{Effect of distributions}

For the performed numerical simulations, particles are given uniform distributions for the starting locations at Inlet 1 and Inlet 2. These uniform distributions are given along both $y$-direction (along the width) and $z$-direction (along the depth), and a normal distribution is assumed on particle size groups to simulate realistic experimental conditions. In this section, distributions along $y-$ and $z$-direction are removed to investigate how the distribution of the starting location affects the separation process. With no distribution on the starting locations, it is assumed that all of the particles start from the midpoint of the channel cross section. However, each particle size group still has normal distributions on their diameter values. In this case, an improvement in separation results is expected since any kind of variation decrease in the processing parameters (such as particle size and/or location) favors the separation performance.

Figure 9a shows the results of the numerical simulation with uniform starting location distribution and normal size distributions with standard deviations defined in Sect. 4.1. In Fig. 9b, the result of the separation without any distribution on $y-$ and $z$-axis is shown (i.e., all of the particles are released from the center of the side channel). Figure 9 shows the separation of the $3-\mu \mathrm{m}$ particles is efficient for

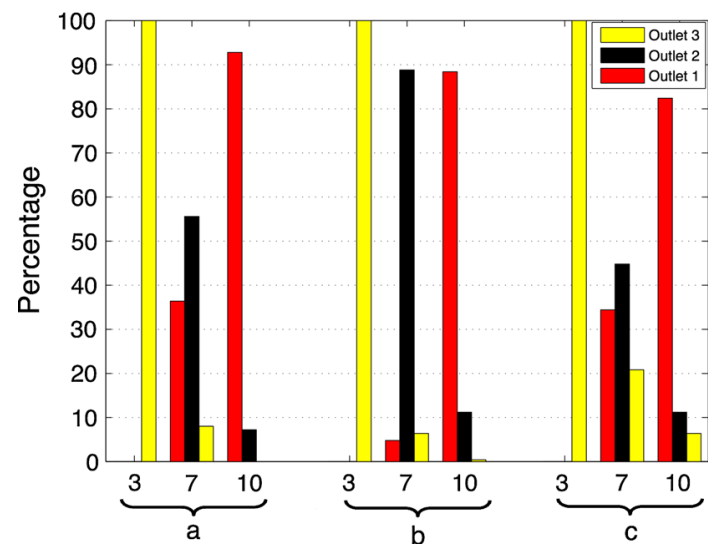

Fig. 9 Bar plots of the separation results: a numerical results from Sect. 4.1, b without $y$ - and $z$-axis distribution, $\mathbf{c}$ double standard deviation
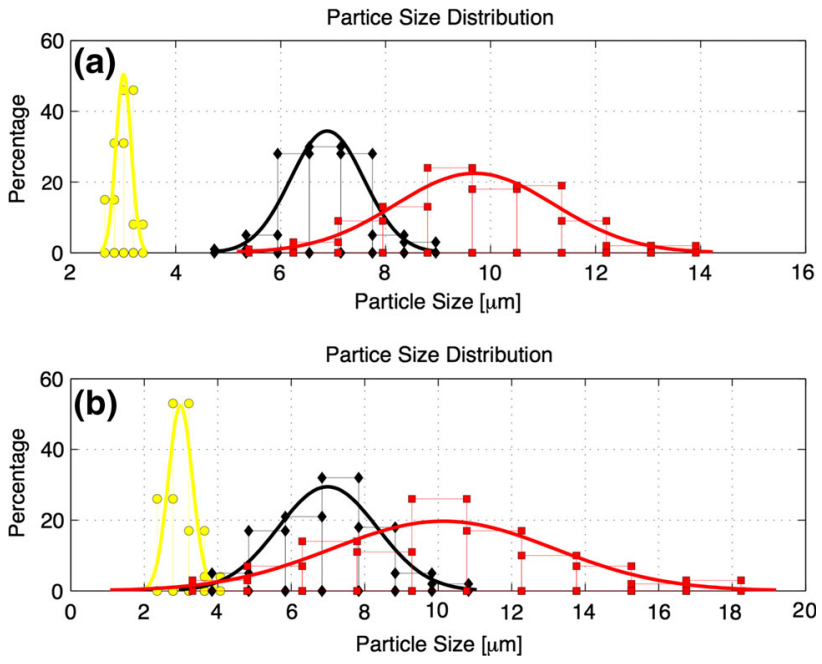

Fig. 10 Size distribution for 3-, 7- and $10-\mu \mathrm{m}$ particles: a distribution used in Sect. 4.1, b distribution with increased standard deviation

any distribution (i.e., in each case all $3-\mu \mathrm{m}$ particles are collected in Outlet 3). Moreover, no significant change on $10-\mu \mathrm{m}$ particles separation occurs as seen from the figure which suggests that the separation of $10-\mu \mathrm{m}$ particles is not significantly affected by the change of the variation of the particles' starting location. On the contrary, the separation efficiency of $7-\mu \mathrm{m}$ particles is significantly affected by the starting location distribution. Without any variation on the starting locations of particles, the separation increases by $\sim 30 \%$ as seen in Fig 9b. In this view, it is clear that any kind of variation decrease in the processing parameters (such as decreased uncertainty on the starting location of the particles) improves the separation efficiency.

In biological systems, the uncertainties in sizes of the cells are significantly larger than the engineered microparticles. To discuss the effect of the larger size variation, the particles with the standard deviation reported in Sect. 4.1 and the 
particles with doubled standard deviation are studied. The size distribution of the simulated particles can be seen in Fig. 10. Figure 10a shows a tight particle size distribution (which is typical for engineered particles), and Fig. 10b shows the size distribution with doubled standard deviation.

Figure 9c shows the separation performance for the particles with doubled size distribution. As seen from the figure, the separation process is significantly impacted from the increased range on particle size. A total of 7- and $10-\mu \mathrm{m}$ particles which exited from the targeted outlet are decreased by $\sim 10 \%$. However, the acoustophoretic force seems to be still not large enough to move even the largest $3-\mu \mathrm{m}$ particles to the center of the channel. Hence, the separation efficiency is still $100 \%$ for $3-\mu \mathrm{m}$ particles. Even this larger distribution may still be a conservative size distribution for actual biological particles. Therefore, a worse separation performance can be expected with actual biological particles. However, with the proposed numerical methodology, the performance of a system can be simulated and the design of the system may be improved to obtain the desired separation performance.

\subsection{Effect of flow rates}

The separation case study in Sect. 4.1 is simulated with two more additional flow rates to understand the effect of the flow rate. For higher buffer flow rate, each outlet flow rate is chosen as 0.25 and $0.04 \mathrm{ml} / \mathrm{min}$ for the side inlet channels. For lower buffer flow rate, each outlet's flow rate is determined as 0.07 and $0.04 \mathrm{ml} / \mathrm{min}$ for the side inlet channels. For each flow rate, an optimum $E_{\mathrm{ac}}$ value is obtained. Higher flow rate means less average amount of time that particles are exposed to acoustic force, so higher $E_{\text {ac }}$ values are needed.

As shown in Fig. 11, the separation performance with high flow rate has decreased about $10 \%$ of $7-\mu \mathrm{m}$ particles.

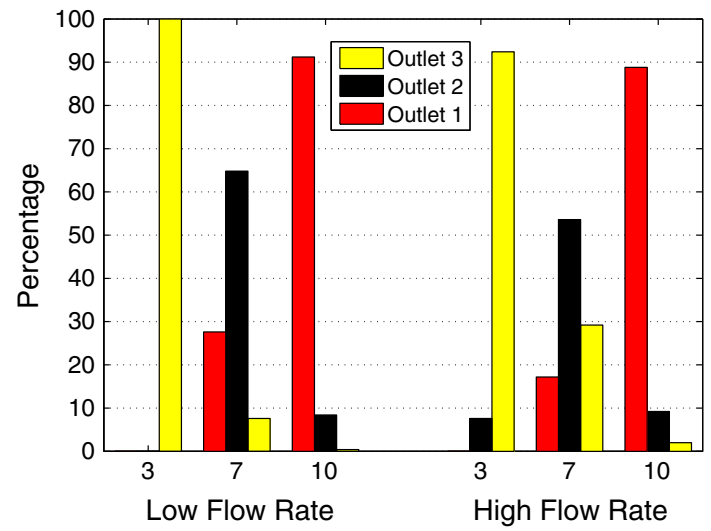

Fig. 11 Bar plots of separation results a with low flow rate $(0.07 \mathrm{ml} /$ min for each outlet and $0.17 \mathrm{ml} / \mathrm{min}$ for buffer inlet), $\mathbf{b}$ with high flow rate $(0.25 \mathrm{ml} / \mathrm{min}$ for each outlet and $0.71 \mathrm{ml} / \mathrm{min}$ for buffer inlet)
The performance has also decreased for 3- and $10-\mu \mathrm{m}$ particles. Optimal $E_{\text {ac }}$ value for high flow rate is also significantly higher than that of the normal flow rate. Separation with lower buffer flow rate has two advantages compared to the separation with higher flow rate. The first one is that particles can be separated with lower $E_{\text {ac }}$ values which leads to lower power consumption. The second advantage is the improvement in separation performance.

\section{Conclusions}

A numerical modeling approach is implemented which simulates the ultrasonic separation of microparticles in a microchannel. The numerical estimations and the experimental results reported in Petersson et al. (2005b, 2007) are in good agreement which shows that several assumptions provided in Sects. 2 and 3 for the numerical simulations can be justified. A numerical simulation tool like this which accounts for statistical distributions in size and starting location can be used to optimize the separator geometrical properties as well as flow rates for a better performance. Moreover, 'what if' scenarios for the design can be evaluated before proceeding with the experimental setup.

In the last section, the factors which affect the separation performance are discussed. Through numerical simulations, it has been shown that the separation performance is negatively affected by the increase in the particle size distributions and the variations in the starting locations of the particles before entering the separation channel. It also has been shown that for the range of parameters used in these analyses, an increase in the buffer flow rate has been shown to decrease the separation performance. To what degree these factors can degrade the separation performance depends on several design parameters of the separator design. Therefore, the proposed numerical methodology may be useful to evaluate and understand the degree of sensitivity of the design to these degrading factors.

There are several assumptions in this study which are aimed to simplify numerical modeling process. One major assumption is the omission of the piezoelectric material. It is thought that the dynamic behavior of the piezoelectric material may affect the acoustic field inside the chip and the channel. The investigation of the effect of dynamic behavior of the piezoelectric material on the particle manipulation in 2D and/or 3D will be one of our scope fields in the future studies.

Another important assumption is the low concentration of microparticles $(\sim 50,000$ particle/ $\mu \mathrm{l})$ as a result of dilution of microparticles and cells. A logical future direction seems to be finite or boundary element modeling of the acoustic field as well as the fluidic field. Such an 
approach will enable numerical simulation of higher concentration of particles. Such finite element analysis approach removes the assumptions of neglecting multiple scattering from particles as well as neglecting distortions in the acoustic standing wave field due to the existence of particles. Through the use of the finite or boundary element modeling for acoustic domain, one can accurately find the acoustic radiation and fluid drag forces on a particle which has a non-spherical shape. It is known that many cell particles (such as red blood cells and platelets) do not have spherical shape but rather a disk-like shape. Such an approach may also enable one to perform similar numerical simulations not only for engineered particles but also nonspherical biological particles. However, the proposed finite element and boundary element approaches do have their own challenges. Considering the separation channel size which is in the order of centimeters, a computation burden would exist for a full 3D analysis. However, relatively simple 2D models may be used as a starter. Moreover, instead of the full length of the separator channel, some portion of the channel may be used to understand the effect of the multiple scattering.

The requirement for low concentration along with the need to prevent high flow rates is the limitation for any application which demands high throughput. Therefore, the acoustophoretic method seems to be more suitable method for medical diagnostic applications since in diagnostic applications the amount of blood that is processed is low and blood can be diluted. The method may not be suitable, at least for the time being, for medical therapeutic applications such as separation of blood cell components for treatment of certain diseases or rare cell separation from peripheral blood such as stem cell therapies. These applications require high throughput and ability to process large volumes of blood which may violate the low concentration and lower flow rate requirements. Dilution of the patients' blood is not possible since it will be transfused back to the patient. The finding in this study indirectly points the need for high throughput methods, such as separation chips with tens (or maybe hundreds) of separation channels in parallel.

Acknowledgments Financial support from the Turkish Scientific and Technical Research Council, Grant No. 112M102, is greatly appreciated.

\section{References}

Adams JD, Soh HT (2010) Tunable acoustophoretic band-pass particle sorter. Appl Phys Lett 97

Bazou D, Castro A, Hoyos M (2012) Controlled cell aggregation in a pulsed acoustic field. Ultrasonics 52(7):842-850

Bhagat lAAS, Papautsky I, (2008) Enhancing particle dispersion in a passive planar micromixer using rectangular obstacles. J Micromech Microeng 18:1-9
Dron O, Ratier C, Hoyos M, Aider J-L (2009) Parametric study of acoustic focusing of particles in a micro-channel in the perspective to improve micro-PIV measurements. Microfluid Nanofluid 7:857-867

Evander M, Johansson L, Lilliehorn T, Piskur J, Lindvall M, Johansson S, Almqvist M, Laurell T, Nilsson J (2007) Noninvasive acoustic cell trapping in a microfluidic perfusion system for online bioassays. Anal Chem 79:2984-2991

Glynne-Jones P, Mishra PP, Boltryk RJ, Hill M (2013) Efficient finite element modeling of radiation forces on elastic particles of arbitrary size and geometry. J Acoust Soc Am 133:1885-1893

Gorkov LP (1962) On the forces acting on a small particle in an acoustic field in an ideal fluid. Sov Phys Doklady 6:773-776

Gralinski I, Alan T, Neild A (2012) Non-contact acoustic trapping in circular cross-section glass capillaries: a numerical study. J Acoust Soc Am 132:2978-2987

Haake A, Neild A, Kim D, Ihm J, Sun Y, Dual J, Ju B (2005) Manipulation of cells using an ultrasonic pressure field. Ultrasound Med Biol 31:857-864

Hawkes JJ, Barrow D, Coakley WT (1998) Micro-particle manipulation in millimetre scale ultrasonic standing wave chambers. Ultrasonics 36:925-931

Hawkes JJ, Barber RW, Emerson DR, Coakley WT (2004) Continuous cell washing and mixing driven by an ultrasound standing wave within a microfluidic channel. Lab Chip 4:446-452

Johnson DA, Feke DL (1995) Methodology for fractionating suspended particles using ultrasonic standing wave and divided flow fields. Separ Technol 5:251-258

Kumar M, Feke DL, Belovich JM (2005) Fractionation of cell mixtures using acoustic and laminar flow fields. Biotechnol Bioeng 89. doi:10.1002/bit.20294

Limaye S, Coakley WT (1998) Clarification of small volume microbial suspensions in an ultrasonic standing wave. J Appl Microbiol 84:1035-1042

Martinez-Duarte R, Gorkin RA III, Abi-Samra K, Madou MJ (2010) The integration of 3D carbone-electrode dielectrophoresis on a CD-like centrifugal microfluidic platform. Lab Chip 10:1030-1043

Nam J, Lee Y, Shin S (2011) Size-dependent microparticles separation through standing surface acoustic waves. Microfluid Nanofluid 11:317-326

Neild A, Oberti S, Haake A, Dual J (2006) Finite element modeling of a micro-particle manipulator. Ultrasonics 44:455-460

Neild A, Oberti S, Dual J (2007) Design, modeling and characterization of microfluidic devices for ultrasonic manipulation. Sens Actuators B 121:452-461

Petersson F, Nilsson A, Holm C, Jnsson H, Laurell T (2005a) Continuous separation of lipid particles from erythrocytes by means of laminar flow and acoustic standing wave forces. Lab Chip 5:20-22

Petersson F, Nilsson A, Jonsson H, Laurell T (2005b) Carrier medium exchange through ultrasonic particle switching in microfluidic channels. Anal Chem 77:1216-1221

Petersson F, Berg LA, Sward-Nilsson A, Laurell T (2007) Free flow acoustophoresis: microfluidic-based mode of particle and cell separation. Anal Chem 79:5117-5123

Settnes M, Bruus H (2012) Forces acting on a small particle in an acoustic field in a viscous fluid. Phys Rev E 85

Shi J, Huang H, Stratton Z, Huang Y, Huang TJ (2009) Continuous particle separation in a microfluidic channel via standing surface acoustic waves (SSAW). Lab Chip 9:3354-3359

Smith DM, Wiggins TA (1972) Sound speeds and laser induced damage in polystyrene. Appl Optics 11:2681

Townsend RJ, Hill M, Harris NR, White NM (2004) Modeling of particle paths passing through an ultrasonic standing wave. Ultrasonics 42:319-324 
Tripp G, Ventikos Y, Taggart DP, Coussios C-C (2011) CFD modeling of an ultrasonic separator for the removal of lipid particles from pericardial suction blood. IEEE Trans Biomed Eng 58:282-290

Trujillo FJ, Eberhardt S, Möller D, Dual J, Knoerzer K (2013) Multiphysics modelling of the separation of suspended particles via frequency ramping of ultrasonic standing waves. Ultrason Sonochem 20:655-666

Yosioka K, Kawasima Y (1955) Acoustic radiation pressure on a compressible sphere. Acoustica 5:167-173 